$\xi=1$ 国

\title{
Survey on QoS metrics and ranking in cloud services
}

\author{
Rajeswari.P ${ }^{1 *}$, Jayashree.K ${ }^{2}$ \\ ${ }^{1}$ Assistant Professor, Rajalakshmi Engineering College \\ ${ }^{2}$ Associate Professor, Rajalakshmi Engineering College \\ *Corresponding author: rajkavin2008@gmail.com
}

\begin{abstract}
The Cloud Computing uses high speed broadband for good Quality of Service (QoS) so that Cloud based application can be used with high speed which entails the minimum response time, less latency rate and reduced amount of loss of packets. Because of the ample range within the delivered Cloud solutions, from the customer's aspect, it's emerged as irksome to decide whose providers they need to utilize and then what's the thought of his or her option. Bestowing suitable metrics is vital in assessing practices. QoS metrics are playing an important role in selecting Cloud providers and also revamping resource utilization efficiency. To guarantee a specialized product is published, describing metrics for assessing the QoS might be an essential requirement. To obtain high quality Cloud applications, Optimal Service Selection is needed. With the increasing number of Cloud services, QoS is usually selected for describing non-functional characteristics of Cloud services. In this paper, a widespread survey on QoS metrics for service vendors and QoS Ranking in Cloud Computing is presented.
\end{abstract}

Keywords: Cloud Computing; Metrics; QoS Ranking; Quality of Service

\section{Introduction}

Cloud Computing is a prominent way of communication which is affecting daily life of the people. The technical and economic advancements take this technology into another direction andit is a suitable way of assessing the on demand resources on the internet through the shared pool such as networks, servers, storage applications, and services [6]. It has proven its acceptability in this current era because of the on-demand capacity management model [1].Cloud Computing has got to appear like a benchmark to obtain demand resources such as Platform, Software, Infrastructure, and so on[5]. Many Cloud Computing operators are accounting good services including Infrastructure-as-a-Service (IaaS), Platform-asa-Service $(\mathrm{PaaS})$ and Software-as-a-Service (SaaS) solutions [7] Cloud Computing have strong features that make it better than the normal computing system like Mobility, More Storage, Easy SetUp, Cheap Service and it is inexpensive comparing to other memory storage[2].

Many companies are using Cloud Computing system presently because the features provided by Cloud application. To achieve high aspect of work, need to ensure about the quality of the services provided to a company [2]. Internet users are increasing day by day, network requirement also increases to obtain good performance. Therefore, many online services demand a very large bandwidth and network performance. Network performance is the element that ferments the users and service providers that should bring new technologies to afford the best services before competitors strike them. QoS is significant topic in Cloud Computing. It is very crucial to make a decision on choosing the Cloud services depending on QoS requirements and it has to be satisfied by both Cloud service providers and Cloud users. QoS refers to the ability of networks to obtain maximum bandwidth and handle other network elements like latency, error rate and uptime. It includes the management of other networks resource by allocating priorities to specific type of data like audio, video and file. It is the ability to afford different priority to different applications, users, or data flows, or to guarantee a certain level of performance.

Basic implementation of QoS needs three major fundamentals such as QoS policy and management functions for controlling end-to-end traffic across network, QoS within one network element, Identification techniques to coordinate QoS from end-to-end between network elements. With the increase of general Cloud offerings, for buyers it is becoming more and more burdensome to choose that vendors can fulfill their QoS needs. Every Cloud provider offers very similar products at completely different costs and capability degrees with various groups of choices, whereas one seller may be inexpensive for memorial solutions, they will be costly for calculation. Moreover, the services on the server side are grasped as a black box to system customers. Therefore, quality evaluation of services before distributing is vital in a Cloud environment. The services need to be examined by QoS metrics to form specified; the services are satisfying the consumer's expectancy. Once assessing Cloud services, a collection of appropriate measurement metrics or criteria should be chosen. In fact, based on the produced research within the analysis of hoary computing devices, the prime of metrics plays a vital role in analyzing implementations [9].

Optimal Service Selection is also quixotic for users to acquire QoS information by evaluating all service candidates by themselves, since conducting real world web service invocations is timeconsuming and resource consuming. Moreover, some QoS properties, for example reliability is difficult to be assessed as longduration observation is required. QoS Ranking provides scalable services and flexible to the diversity of end users. The active user and training users are identified and the similarity between those two users are calculated either Kendall Rank Correlation Coefficient or Pearson Correlation Coefficient. 
The aim of this paper is to present the survey of determining QoS metrics that could be applied by the service manufacturers and to evaluate the services that effectuate the consumer's priority and QoS ranking. The remainder of this paper is organized as follows: Section 2 provides a survey on QoS metrics. Section 3 provides a survey on QoS ranking. Section 4 concludes the paper.

\section{Metrics for cloud services evaluation}

QoS is defined as a set of properties including response time, throughput, availability, reputation, failure probability, etc [3]. Among these QoS properties, values of some properties such as response time, user-observed availability, etc. are essential to be measured at the client-side [10]. It is impractical to get such QoS information from service providers, since these values are susceptible to the uncertain Internet environment. Therefore, different users may observe quite different QoS values of the same Cloud service.

QoS presents the non-functional characteristics of the Cloud services. The dynamic Cloud services consist of five generic QoS properties viz execution price, execution duration, reliability, availability, and reputation. The flexible processes, includes adaptive service composed of execution time, availability, price, reputation, and data quality. An efficient service composition approach considers both generic and domain-specific QoS properties. Various QoS measurement of Cloud services has been introduced in the SLA, such as IBMs WSLA technique and the work from HP [8]. Data security, integrating applications into different systems, Service level Agreements and the management tools can enhance it. Management methods can control the high programmability of the peripheral resources in the Cloud system for effective QoS [11].

Factors driving adoption of Cloud Computing "Overall IT cost reduction, reduced risk of IT disruption from external factors such as natural disasters, greater overall business agility and flexibility, enhanced IT infrastructure efficiency and faster deployment time"[12]. Cloud Computing is an evolving paradigm [4]. The NIST definition characterizes important aspects of Cloud Computing and is intended to serve as a means for broad comparisons of Cloud services and deployment strategies, and to provide a baseline for discussion from what is Cloud Computing to how to best use Cloud Computing. The service and deployment models defined form a simple taxonomy that is not intended to prescribe or constrain any particular method of deployment, service delivery, or business operation[13].There are three aspects of Cloud services such as Economics, Security and Performance and these metrics are discussed below.

\subsection{Economics Metrics}

It is to compare and to check totally different costs of services as they furnish variety of options and so have got several dimensions. However, an equivalent supplier offers completely various Virtual Machines that can meet users' requirements. Economics has been typically considered a driving factor in the adoption of Cloud Computing. Berkeley's view of Cloud Computing is factored by elasticity and price [14]. Thus, we collected and organized relevant metrics for these two properties respectively.

\subsubsection{Cost}

An elastic Cloud service provides the growth and reduction of workload, and drastically improves the speed of response to modified workload.

\subsubsection{Elasticity}

Moving computing process to the Cloud incurs a lot of cost.

\subsection{Security Metrics}

Information security and privacy are crucial problems for almost each one company. Sharing data underneath a different organization's administration is usually places significant concerns under threat. For instance, economic institutions generally need compliance with the laws regarding data integrity and also security. The protection of commercial Cloud services has got many dimensions and challenges which individuals must be worried about $[14,15]$. Even so, not many safety feedbacks were mirrored in the famous major reports. Security and Safety are multi-dimensional and also contains numerous characteristics similar to saving trust and safety, data integrity and availability. The features of security metrics are discussed below.

\subsubsection{Data Security}

It represents a vast group of plans, systems, and also equipment is utilized to guard programs, as well as the related infrastructure of Cloud systems.

\subsubsection{Authentication}

The process of determining, whether or not to access the feature is said to be authentication and it usually involves verifying the validity of a minimum of one type of identification.

\subsection{Performance Metrics}

There are several unique options provided by Cloud vendors dealing with the IT needs of multiple companies. Each decision has got very different expertise regarding performance, service latency and precision. Institutions need to recognize however their programs can do on the numerous Clouds and also whether or not those deployments satisfy their goals. Performance means diverse things in many contexts. It's defined by the metrics such as response time that is the time could it require to process a demand, throughput is the measure of how many units of information a system can process in a given amount of time, or even timeliness is the capability to meet deadlines. Many aspects of performance characteristics identified from the previous major researches could be summarized below.

\subsubsection{Communication}

It specifies the data transfer between internal service instances or between external consumer and also the Cloud.

\subsubsection{Computation}

It indicates the computing data or task processing in the Cloud systems.

\subsubsection{Memory}

Designed for rapid use of temporary storage of information that may be attained from slow-accessed hard disk drive.

\subsubsection{Time}

Project completion within time while maintaining quality is very important for the success of projects.

\subsection{General Metrics}

Cloud service is known as a process resource or a network path or a middleware service or a Cloud application. Within the Cloud, the services usually are distributed across totally different suppliers and these features are important for service suppliers. To target 
QoS metrics of Cloud services that may be monitored and measured. The classifications of chosen general metrics are discussed below.

\subsubsection{Reliability}

It is the power of a service to remain functional with time without malfunction. The capability of the service to keep on operating with a certain degree of efficiency constantly.

\subsubsection{Efficiency}

The resources occupied for services when providing the wanted operation and it evaluates performance of web services that employs the sources.

\subsubsection{Adaptability}

The level of optimizing the solutions for the utilization of every service based software.

\subsubsection{Scalability}

It represents the potentiality of boosting the calculating power of the service provider's program, as well as the product's ability to process a number of client's demands at a certain time period.

\subsubsection{Reusability}

It is known as the level to which an application component or even other work system may be used in a number of program or application

\subsubsection{Availability}

Availability refers to the ability of a user to access information or resources in a specified location and in the correct format.

\section{QoS ranking techniques}

QoS is describing non-functional characteristics of Cloud services. Among its different properties of Cloud services, few are userdependent and have different values for different users. Example is response time, in-vocation failure probability, etc. [3]. Rankingbased QoS prediction proceeds aiming at predicting the quality ranking of the target Cloud services instead of the detailed QoS values.

Breese et al. [16] have proposed merging filtering algorithms that predict the value of items to a particular user based on a database of user votes from a sample or population of other users. They use two basic classes of evaluation metrics. The first specifies accuracy over a set of individual predictions in terms of average absolute deviation and the second determines the utility of a ranked list of suggested items. Bayesian networks generally have smaller memory requirements and allow for faster predictions than a memory-based technique such as correlation, but Bayesian methods examined here require a learning phase that can take up to several hours and results are reflected in the recommendations.

Linden et al. [17] proposed Recommendation Algorithm which decides a set of customers whose purchased and rated items overlap the user's purchased and rated items. The algorithm clumps items from these similar customers, eliminates items the user has already purchased or rated, and recommends the remaining items to the user. It initiates high quality recommendations and the algorithm must respond immediately to new information and it is used to differentiate the online store for each customer, but it needs to apply recommendation algorithms for targeted marketing, both online and offline.
Yu et al. [18] have proposed a broker-based architecture to assist the selection of QoS-based services. The aim of service selection is to maximize an application-specific utility function under the end-to- end QoS constraints. The combinatorial model explains the problem as a multidimensional multichoice 0-1 knapsack problem. The graph model defines the problem as a multiconstraint optimal path problem. QoS for web services directs to various nonfunctional characteristics such as response time, throughput, availability, reliability and failure probability.

Saurabh Kumar Garg et al. [19] have proposed a framework to measure the quality and prioritize Cloud services. This framework makes major effect and creates healthy competition among Cloud providers to satisfy their Service Level Agreement (SLA) and improve their QoS. They proposed an Analytical Hierarchical Process (AHP) based ranking mechanism which can determines the Cloud services based on different applications depending on QoS requirements. This technique is used only for quantifiable attributes such as Accountability, Agility, Cost, Assurance of Service, Performance, Security, Privacy, and Usability. It is not suitable for non-quantifiable attributes such as Service Response-time, Sustainability, Accuracy, Interoperability, Availability, Reliability and Stability.

Zibin Zheng et al. [20] have proposed Cloud Rank approaches to rank the Cloud services in an optimal way using greedy algorithm. It ranks the component instead of service, but this algorithm is used to rank a set of items, which considers the explicitly rated items and the unrated items equally. It does not assure that the explicitly rated items will be ranked correctly.

The rating-based QoS prediction approaches aim at predicting its values for different service users. Zibin Zheng et al. [21] have proposed a Cloud Rank Prediction Framework that speculates the Ranking directly instead of predicting the experimental QoS values and the predicted values can be involved to rank the target Cloud services. The major problem of making QoS-driven Cloud service quality ranking is that the Cloud service quality ranking of a user cannot be transferred directly to another user, since the user locations are different. So, the author proposed a personalized QoS Ranking for Cloud services and determines all the Cloud services at the user-side and rank the Cloud services based on the observed performance. Moreover, it is difficult for the service users to evaluate all the Cloud services themselves, since there may occur a huge number of Cloud services in the Internet.

Location-aware Web service recommender system named LoRec, which involves both web service QoS values and user locations for making personalized QoS prediction. Users of LoRec share their past usage experience of web services, and the system affords personalized service recommendations to them. Therefore, different users may perceive quite different values of the same web service. It is also impractical for users to acquire QoS information by evaluating all service candidates by themselves, since conducting real world Web service invocations is time-consuming and resource consuming.

\section{Conclusion}

Cloud is now a crucial exemplar for outsourcing diverse computer needs of institutions. To select suitable between several Cloud companies, clients ought to have the method to recognize and also assess crucial performance standards, which are necessary to their programs. The choice of metrics has been known as being essential within the evaluation of computer systems. Optimal service selection is important to obtain high quality Cloud applications. Cloud Rank Framework provides the same quality. An optimal VM allocation is used to improve the quality of Cloud applications. In this paper, we have carried out a significant review in QoS Ranking of Cloud services and metrics. This survey paper will expectantly inspire future researchers to come up with high quality Cloud applications using QoS ranking techniques and metrics. 


\section{References}

[1] Prerita G, Harmunish T, Gagandeep S B , Quality of services in cloud computing: issues, challenges and analysis, International Journal of New Innovations in Engineering and Technology, Vol. 3,No. 3, (2015), pp:76-82

[2] Hashem Ramadan H, Divya K, Quality of service (qos) in cloud computing, International Journal of Computer Science and Information Technologies, Vol. 8 (3), (2017), pp:318-320

[3] Subha M, Uthaya Banu M, A survey on qos ranking in cloud computing, ISSN 2250-2459, ISO 9001:2008 Certified Journal, Vol 4, No. 2,(2014) Website: www.ijetae.com

[4] Poonam K, Satav S D, A survey paper on quality of service in cloud computing, International Journal of Science and Research, Vol. 4, No.11, (2015), pp: 2119-2122

[5] Amid K B,Seyyed M H, QoS metrics for cloud computing services evaluation, I.J. Intelligent Systems and Applications, Vol.12, (2014), pp:27-33

[6] Srinivas J, VenkataSubba Reddy K, MoizOyser A, Cloud computing basics, International Journal of Advanced Research in Computer and Communication Engineering, Vol. 1, No. 5, (2012), pp: 343-347

[7] Zhang Q, Cheng L, Boutaba R, Cloud computing: state-of-the-art and research challenges, J Internet ServAppl (2010)

[8] Zhang S, Zhu C, Sin J K O \& Mok P K T, A novel ultrathin elevated channel low-temperature poly-Si TFT, IEEE Electron Device Lett., Vol. 20, (1999), pp: 569-571

[9] Obaidat M S, Boudriga N A, Fundamentals of performance evaluation of computer and telecommunication systems, Hoboken, New Jersey: John Wiley \& Sons, Inc. (2010)

[10] Thio N, Karunasekera S, Automatic measurement of a QoS metric for web service recommendation, in Proceedings Australian Software Engineering Conference, (2005), pp. 202-211.

[11] Wu J, Chen L, Feng Y, Zheng Z, Zhou M \& Wu Z, Predicting QoS for service selection by neighborhood based collaborative filtering, IEEE Trans. System, Man, and Cybernetics.

[12] http://www.thewhir.com/web-hosting-news/Cloud-spending-increasesamong-new-zealand-organizations-driven-saas-applications-study

[13] Mell P, Grance T, The NIST definition of cloud computing. National institute of standards and technology, Information Technology Laboratory, Vol. 15, (2009)

[14] Armbrust M, Fox A, Griffith R, Joseph A D, Katz R \& Konwinski A, A view of cloud computing, Commun. ACM, 53 (4) (2010), pp. 50-58

[15] Wang J, Zheng X, Luo D, Sensitive Data Protection Based on Intrusion Tolerance in Cloud Computing, International Journal of Intelligent Systems and Applications (IJISA), 3(1), (2011), pp 58-66

[16] Breese J S, Heckerman D, Kadie C, Empirical analysis of predictive algorithms for collaborative filtering, Proc. 14th Ann. Conf. Uncertainty in Artificial Intelligence (UAI '98), (1998), pp: $43-$ 52

[17] Linden G, Smith B, York J, Amazon.com Recommendations: Item-to-Item Collaborative Filtering, IEEE Internet Computing, Vol. 7, No. 1, (2003), pp. 76-80

[18] Yu T, Zhang Y, Lin K J, Efficient algorithms for web services selection with end-to-end QoS constraints, ACM Trans. Web, Vol. 1, No. 1, (2007), pp. 1-26

[19] Saurabh Kumar G, Versteeg S , RajkumarBuyya, SMICloud: A framework for comparing and ranking cloud services, Published in Fourth IEEE International Conference on Utility and Cloud Computing (2011)

[20] Zheng Z, Zhang Y, Lyu M R, CloudRank: A QoS driven component ranking framework for cloud computing, Proc. Int'l Symp. Reliable Distributed Systems (SRDS'10), (2010), pp. 184-193

[21] Zheng Z, Wu X, Zhang Y, Lyu M R \& Wang J, QoS ranking prediction for Cloud services, IEEE Transactions on Parallel and Distributed Systems, Vol. 99, (2012).

[22]R. Farah Sayeed, S. Princey, and S. Priyanka. "Deployment of MultiCloud Environment with Avoidance of DDOS Attack and Secured Data Privacy." International Journal of Applied Engineering Research 10, no. 9 (2015): 8121-8124. 